\title{
La fidélité du consommateur entre effet d'usage, effet de cycle de vie et effet de marque
}

\section{Dominique Desjeux}

\section{(2) OpenEdition}

12 Journals

Édition électronique

URL : http://journals.openedition.org/communicationorganisation/3240

DOI : 10.4000/communicationorganisation.3240

ISSN : $1775-3546$

Éditeur

Presses universitaires de Bordeaux

Édition imprimée

Date de publication : 1 juin 2005

Pagination : $69-58$

ISSN : 1168-5549

\section{Référence électronique}

Dominique Desjeux, «La fidélité du consommateur entre effet d'usage, effet de cycle de vie et effet de marque », Communication et organisation [En ligne], 27 | 2005, mis en ligne le 07 juin 2012, consulté le 04 mai 2019. URL : http://journals.openedition.org/communicationorganisation/3240 ; DOI : 10.4000/ communicationorganisation.3240

Ce document a été généré automatiquement le 4 mai 2019.

(c) Presses universitaires de Bordeaux 


\title{
La fidélité du consommateur entre effet d'usage, effet de cycle de vie et effet de
}

\section{marque}

\author{
Dominique Desjeux
}

1 A la fin d'un livre ouvert et complet sur Le comportement du Consommateur et de l'acheteur (2003), Richard Ladwein présente les différentes thèses actuelles sur la fidélité de l'acheteur. Il rappelle que la fidélité relève d'une explication multifactorielle, qu'elle ne se réduit pas à l'achat répété ni à la satisfaction mais qu'elle intègre aussi une dimension identitaire, émotionnelle et expérientielle, dimensions qui ont été développées plus généralement par Oliver Badot, Bernard Cova ou Patrick Hetzel par ailleurs. Globalement la fidélité, vue du point des sciences de gestion, du marketing et de la psychologie de la consommation, se centre sur la marque, sur le moment de l'achat et sur les processus d'arbitrage micro-individuels. La marque a le plus souvent un statut de variable indépendante.

2 Par contre, si je change d'échelle d'observation (D. Desjeux, 2004, Les sciences sociales) et si je prends un point de vue anthropologique et que je passe à une échelle micro-sociale, je découvre que la marque change de statut explicatif. Elle devient une variable dépendante des pratiques et des occasions qui déclenchent le processus d'acquisition marchand ou non marchand des biens de consommation, des étapes du processus socio-économique qui part de la recherche-développement en entreprise, en passant par le marketing et le merchandising, pour aller jusqu'aux usages et aux rangements dans l'espace domestique.

Ce changement d'échelle permet de faire apparaitre de nouvelles facettes de l'explication de la fidélité du consommateur vis-à-vis de la marque. Sans contester l'importance de la marque dans le champ d'une filière économique ou à l'étape du « réenchantement » ou de «transsubstantiation» (D. Desjeux, postface, 2004) du bien de consommation par le marketing, il la relativise cependant. L'échelle micro-sociale permet de mieux faire apparaître à la fois la place du produit et son autonomie relative par rapport à la marque, 
le poids des effets d'appartenance sociale et le lien entre la dynamique de la variabilité des comportements et les effets de structure liés aux étapes du cycle de vie.

À l'échelle d'observation micro-sociale, comprendre la place de la marque et de la fidélité demande de faire une distinction anthropologique clé: distinguer le bien de consommation qui relève d'une logique d'usage utilitaire ou esthétique, de la marque qui relève soit d'une logique cognitive au moment de l'achat, soit d'une logique symbolique qui s'appuie sur les grands imaginaires sociaux au moment de l'étape publicitaire. Le constat est que le lien entre usage et pratique d'un côté et imaginaire et représentations de l'autre ne va pas de soi. L'échelle micro-sociale fait apparaître qu'entre les pratiques et l'imaginaire se glissent les contraintes du jeu social et celles des effets d'appartenance ou de distinctions sociales, et ceci tout au long des différentes étapes du cycle de vie. Ces contraintes, ces effets de structure et les normes sociales qui leurs sont associées expliquent l'écart fréquent entre ce qui est dit, ce qui est pensé et ce qui est décidé par le consommateur et par là une partie de la fidélité ou de l'infidélité.

5 À cette échelle, la volonté et le libre arbitre des acteurs sont moins visibles que les effets de situation, de jeu social ou de facteur sociétal. Vers 1992, par exemple, au moment où le pouvoir d'achat a fortement baissé en France, le comportement des consommateurs est devenu plus «volatile». Le consommateur est peut-être devenu "plus malin», plus « stratège » ou plus « manager » comme individu, pour reprendre des termes de l'époque dans les journaux, mais je peux aussi faire l'hypothèse que le consommateur a toujours été « malin ». C'est sous contrainte de budget et de revenu qu'il a dû adopter un nouveau comportement. Un autre indice qui conforte cette hypothèse de l'importance des contraintes et que c'est aussi à partir du début des années quatre vingt dix que ce sont développées en France de nouvelles formes de distribution plus adaptées à la consommation des populations les plus démunies en expansion à cette même période. C'est un moment de croissance des «hard discounters » et du crédit à la consommation lequel sera associé au phénomène de surendettement et de renouvellement de la profession d'huissier ${ }^{1}$. L'hypothèse est que l'instabilité ou la variabilité de la vie sociale est un facteur explicatif important de l'infidélité éventuelle du consommateur.

Dans cet article je pars d'une série d'enquêtes qualitatives réalisées depuis une dizaine d'années avec Sophie Taponier (1998), Sophie Alami (2004), Isabelle Garabuau-Moussaoui (2002), et un réseau de chercheurs et d'étudiants sur la variation du lien entre l'objet, ou le bien de consommation, la marque et les effets de cycle de vie. La principale conclusion est que les étapes, ainsi que les moments de passage entre ces étapes sont des déclencheurs important d'occasions d'achat et de changement de produits au-delà des seuls effets de marque. C'est ce que je vais essayer de montrer dans le cas des jeunes qui sont tout spécialement sensibles aux effets du cycle de vie appliqués ici au cas de la consommation d'alcool et tout particulièrement à celle du pastis(Desjeux et alii, 1994).

\section{Des Lolita aux Tanguy : La jeunesse, une phase instable de la vie en train de s'allonger et de plus en plus multiculturelle}

7 La jeunesse est une catégorie qui peut aller suivant les analyses de 11 à 30 ans. C'est une catégorie aux frontières mouvantes historiquement et culturellement. Elle s'allonge aujourd'hui autant du côté de l'enfance, avec les « Lolita » (J.M. Normand, 2004), que du 
côté de la vie adulte avec l'allongement des études et le phénomène dit des "Tanguy ", ces jeunes qui restent chez leurs parents jusqu'à 30 ans. Pour l'INSEE en 2000 est jeune une personne ayant de 16 à 29 ans. Pour Médiamétrie cette catégorie couvre les personnes de 13 à 25 ans.

8 Les jeunes sont aussi une catégorie très hétérogène (I. Garabuau-Moussaoui, 2004) puisqu'elle comprend des étudiants, des "CDD", des "Petits boulots" et des chômeurs, chômage qui touche autour de 25 \% des jeunes en 2005. Ils comprennent des jeunes filles avec ou sans enfant, des " hétéros » ou des «homos", des jeunes qui ne votent pas et à l'inverse des militants convaincus notamment contre la consommation et la pub (J. Chwarzciannek, 2004), des jeunes des cités issus de l'immigration et des jeunes des « beaux quartiers », etc.

9 Une forte caractéristique de la jeunesse en est l'aspect très multiculturel et pluriethnique. D'après l'INSEE comme l'indique le directeur des programmes de Skyrock, seuls 6,2 \% des 60 ans et plus sont issus de l'immigration en 1ère ou 2ème génération, alors que $23,2 \%$ des moins de 18 ans sont issus de l'immigration en 1ère ou 2ème génération. Il faudrait juste se demander de quelle immigration il s'agit : africaine, maghrébine ou autre.

Enfin l'importance des groupes de pairs et par là des réseaux y est prépondérante dans le fonctionnement du lien social entre jeunes.

De façon approximative il est possible d'organiser les étapes des cycles du début de la vie en trois grandes phases dont les frontières et la façon de les nommer restent malgré tout instables. Elles suivent en gros les étapes du système scolaire : l'enfance jusque vers 11 ans ; l'adolescence (entre 11/12 ans et 16/18 ans) ; la jeunesse (entre 16/18 ans et 25/30 ans) ; puis l'entrée dans l'étape adulte au moment de la mise en couple, de l'arrivée d'un enfant et l'entrée dans la vie active, pour reprendre les trois critères macro-sociaux définis par Olivier Galland (1991). Celle-ci tend à se raccourcir du point de vue du critère du travail puisque seul $30 \%$ d'une classe d'âge travaille encore à 60 ans aujourd'hui (cf. X Gaulier, 1988 ou A.M. Guillemard, 2003).

Ces étapes sont elles-mêmes en partie structurées par l'école depuis la maternelle jusqu'à l'université que les jeunes y aient été inclus ou exclus. La jeunesse se développe entre deux pôles : les étapes du système scolaire d'un côté et celle de la vie professionnelle de l'autre. Elles s'organisent aussi autour d'autres clivages liés à la mobilité, aux genres et au revenu: avoir ou non un permis de conduire (J.P. Orfeuil, 2004), avoir ou non l'autorisation des parents pour sortir, - autorisation plus restrictive pour les filles comme l'a montré Laurence Buffet - (in M. Bonnet, à paraître), avoir une capacité budgétaire forte ou faible, etc. Ces clivages liés à la mobilité sont très structurants de la capacité que les jeunes auront ou non à avoir accès à tel ou tel lieu de distribution et tout particulièrement à ceux des centres villes.

Finalement, la jeunesse au sens large apparaît comme un moment du cycle de vie plutôt dominé par la variabilité des opportunités de mobilité et de situations sociales et culturelles. Cette variabilité des comportements est aussi souvent liée à l'instabilité de l'emploi et du chômage, voire à l'instabilité de la famille du fait des divorces. C'est cette instabilité forte à cette étape du cycle de vie qui semble influencer le choix des biens de consommation et leur variabilité ou leur « volatilité ». 


\section{Les jeunes et la consommation d'alcool}

14 Avec la consommation d'alcool des jeunes nous allons essayer de mieux comprendre le lien entre le cycle de vie, les occasions d'usage et le positionnement du produit par rapport à la marque. En France, il existe en gros trois grandes occasions sociales de boire de l'alcool que l'on soit jeune ou adulte. La première est liée à l'apéritif qui se prend plutôt le soir, pendant les week-ends et plus généralement pendant les temps de détente informelle, certaines de ces occasions étant fortement liées à l'été et aux vacances. La deuxième occasion est liée aux fêtes et aux grands événements familiaux ou amicaux. La troisième est plus indéfinie, elle est liée à la recherche de l'ivresse. Ces trois occasions structurent les usages du pastis ce qui fait que le pastis apparaît comme un alcool parmi d'autres, qu'il n'est pas considéré comme un alcool de fête exceptionnelle au contraire du champagne et qu'il n'est pas considéré comme un alcool de transgression liée à la nuit, comme un alcool de «cuite». C'est donc un alcool de conformité sociale, en vue d'entretenir la sociabilité de jour.

\section{Une socialisation ambivalente de $10 / 12$ ans à $18 / 19$ ans : entre la conformité entre pairs et la transgression familiale}

15 Notre enquête qualitative de 1995, et cela semble bien confirmé aujourd'hui par les enquêtes citées dans la bibliographie, montre qu'au moment de l'adolescence une partie des individus deviennent membres "actifs" de la communauté familiale adulte. Il s'agit d'un processus d'intégration des jeunes adolescents à la communauté des adultes qui est fait à la fois d'adhésion et d'opposition (V. Ciccheli, 2001). Ils acquièrent en parallèle de plus en plus d'autonomie par rapport à leurs parents grâce à la fréquentation de leurs pairs. L'alcool, comme objet social (I.Garabuau-Moussaoui, D. Desjeux, 2003), va jouer un rôle important dans ce processus de socialisation à la fois comme objet complice entre générations, comme objets de la distanciation familiale et de l'intégration entre pairs, comme objet de transgression entre pairs et entre genres et enfin comme objet de conformité au groupe de pairs. Cette conformité entre à son tour dans la construction de l'identité de genre, hétéro ou homo, notamment.

L'alcool apparaît donc comme un objet de socialisation de jour et de nuit en fonction de ses différents usages sociaux. Pendant la journée il va jouer soit sa fonction de complicité intergénérationnelle à la maison avec l'apéritif ou au café, soit sa fonction de distanciation familiale avec une consommation entre pairs au bar avec les copains ou à la maison mais sans les parents. La nuit, l'alcool va jouer son rôle de transgression d'un côté et celui de conformité au groupe de pair de l'autre ce qui conduit à une sorte « d'interdit» de l'abstinence pour les garçons, une importance plus grande accordée aux cocktails et aux alcools forts et une recherche de la « cuite ", tout cela sous contrainte de coût ce qui expliquerait en partir l'intérêt pour la bière bon marché.

La place du pastis et de la marque, Pernod ou Ricard, apparaît donc comme fortement structurée par ces règles de la socialisation. Ainsi le pastis est exclu de la nuit, des discothèques et de la transgression. Il est l'un des éléments courants des apéritifs familiaux. C'est pourquoi dans l'imaginaire des jeunes les marques de pastis ne semblent pas avoir de fonction forte de différenciation ou de distinction sociale. Le pastis et les marques qui lui sont associées appartiennent donc au registre de la continuité et de la 
conformité de jour, loin de la nuit, du contournement des codes sociaux et des tribus, inspirées de Michel Maffesoli (M. Maffesoli, 1985), pour le whisky de la marque Clan Campbell à la même époque.

\section{La transition de 18 à 30 ans : un éclatement des modes de vie}

18 Après 18 ans, à la sortie du secondaire, ou un peu avant pour ceux qui sortent plus tôt du système scolaire, pendant la période où les jeunes ont quitté l'adolescence mais où ils n'ont pas encore une vie très stabilisée, la jeunesse se divise en plusieurs modes de vie. Nous en avons choisis trois: les étudiants, les jeunes salariés célibataires, les jeunes salariés en couple.

La vie étudiante se caractérise par une dépendance financière qui limite la consommation. L'étudiant dépend de l'aide familiale et du revenu des " petits boulots». C'est le moment de l'apprentissage de la liberté. La solidarité entre copains et amis remplace en partie la solidarité primaire liée à la famille proche. Ces réseaux peuvent être très instables en fonction des affinités et de la mobilité scolaire de chacun. L'instabilité des réseaux entraîne une instabilité des modes de consommation des produits et des marques. À la maison, le pastis continue à être l'une des boissons dédiée aux rencontres informelles entre amis. Au café la bière et le pastis traditionnels sont associées à la recherche de nouveaux goûts et donc à une diversification des alcools.

Les jeunes salariés cherchent à quitter leurs parents et à accéder à l'indépendance. C'est à la fois une vie de célibataire et une transformation de la vie familiale en réseau avec les frères, les sœurs et les copains-voisins. C'est un peu chacun chez soi mais tous ensemble. La période despremiers emplois est aussi une période de changements. Ceux qui ont vécu leur jeunesse pendant les "trente glorieuses" ont trouvé un emploi assez rapidement.Les autres, plus jeunes, ont connu les petits boulots, le chômage et donc des changements fréquents d'emploi. Cette période est placée sous le signe du changement et de l'instabilité professionnelle. La place du pastis et de la marque commence à changer. Certains découvrent le pastis. D'autres associent le pastis à un "alcool de pauvre», à un alcool pour d'autres, à une distinction sociale négative et donc l'abandonnent. L'expérience de l'alcool se diversifie grâce à de nouveaux alcools mais surtout avec « l'apparition » du vin. La pratique du vin semble associée au passage vers la vie adulte et vers l'autonomie. Pour certains, la marque devient plus importante soit comme signe d'appartenance communautaire régionale, soit comme signe de qualité. Pour d'autres cependant la marque n'a pas d'importance : l'important c'est d'aimer.

21 Le mode de vie des jeunes couples salariés se caractérise par la mise en place d'une nouvelle stabilité, par l'arrivée de nouvelles normes. Pour certains, le repas est vécu comme une occasion de créer un nouvel ordre de la boisson avec un apprentissage plus systématique de la consommation de vin, de digestifs et d'apéritifs. Le pastis change de positionnement. Quand il y a consommation de pastis elle se pratique dans l'espace domestique intime. L'alcool et le vin changent aussi de positionnement. Ils sont associés à des marqueurs de distinction sociale. La marque, l'étiquette, la forme de la bouteille jouent un rôle plus important au moins dans l'espace domestique intime. 


\section{Le pastis dans les étapes du cycle de vie}

22 Le cas de l'alcool en général et du pastis en particulier est un bon exemple du lien entre l'usage d'un produit, sa codification sociale entre ce qu'il est prescrit, permis ou interdit de boire, l'importance variable du produit ou de la marque et la variabilité des comportements d'achat. Ainsi, dans les premières étapes du cycle de vie, le pastis symbolise les moments informels entre pairs et la sociabilité de jour public ou privée. Le pastis n'apparaît pas comme un fort marqueur social. Dans la dernière partie du cycle de la jeunesse, la dimension stratégique de la marque dans le jeu social apparaît plus forte. Le pastis peut être alors soit dévalorisé socialement et abandonné, soit remplir une nouvelle fonction identitaire dans laquelle la marque, Ricard ou Pernod notamment, pourra jouer un rôle plus important.

Cependant, le lien entre cycle de vie, produit et marque n'est jamais donné une fois pour toute. Il est sans cesse reconstruit. Ceci demande d'observer les nouvelles sources de l'instabilité sociale comme les séparations familiales, le chômage, la mobilité liée aux migrations internationales, voire les phénomènes de contestation de la consommation et des marques. Cette contestation semble tout particulièrement s'exprimer pendant la période de transition entre la fin de l'adolescence et le moment de la socialisation professionnelle. Elle participe peut-être d'une forme de résistance à cette socialisation et $\mathrm{au}$ « nouvel ordre du corps » qui s'instaure au moment d'intégrer la vie professionnelle comme nous allons le voir avec les vêtements et le «look» du corps.

\section{L'entrée dans le monde du travail : abandonner tout ce qui fait « jeune » et « désordre »}

24 Avant d'entrer dans le monde du travail, les jeunes utilisent deux sortes de marquages (d'après A Guilot, 2004): les marques sociales modulables comme le vêtement, les cheveux ou la barbe, le piercing, les accessoires (bijoux, casquettes puis chapeaux, ceintures, chaussures, objets de la communication: téléphones, agendas, $\mathrm{Hi} \mathrm{Fi,}$ ordinateurs, etc.) et les marques indélébiles comme le tatouage. En entrant dans le monde du travail les marquages vont évoluer et notamment les marquages modulables.

Cela va se traduire par l'abandon de tout ce qui fait jeune ou adolescent et donc "désordre ». Les cheveux de très longs deviennent courts et la «boule à zéro » tend à disparaître. Certains garçons se laissent pousser la barbe ou un bouc pour faire plus vieux ou plus virils. La casquette est abandonnée. Pour les filles, c'est la fin des couettes et des cheveux teintés. C'est aussi le passage au maquillage discret. Pour les hommes cela peut se traduire par un passage au maquillage.

Pour tous, les vêtements deviennent "classiques»: les jeans sont remplacés par des pantalons de fille ou des tailleurs. Les baskets, les piercings visibles disparaissent. Cependant une partie de ces abandons pourront revenir le week-end ou les «casual fridays ".

27 Si dans le domaine de l'habillement et du look, l'adolescence est l'âge où la marque est plutôt mise en valeur, montrée et exhibée. En sortant de la jeunesse la marque se fait plus discrète pour tout ce qui touche à l'alimentation, les vêtements et les objets de communication (cf. M. Bouchardeau et alii, 2004). 
La discrétion de la marque permet de gérer la période d'incertitude liée à la sortie de la jeunesse et tout spécialement l'entrée dans le monde du travail. Une marque discrète n'est reconnue que par les initiés. Cela répond au besoin d'appartenance. Et en même temps ne pas montrer de marque permet de ne pas se singulariser et donc protège sa singularité. La discrétion du rapport à la marque permet à la fois de montrer que l'on est sorti du désordre et que l'on est conforme à l'ordre social requis pour trouver du travail tout en signifiant aux autres que l'on reste unique.

\section{Conclusion : variabilité des situations sociales et variabilité du rapport à la marque}

Plus généralement, pour les jeunes le lien avec les marques est un rapport instable. Le rapport aux marques est très sensible aux changements de cycle scolaire. L'autonomisation par rapport aux parents influe sur le choix des marques du fait de l'importance de la norme du groupe de pairs qui influence le choix de la marque. Mais le rapport aux marques peut devenir instable en fonction de la fréquence de changement de groupes de pairs. Il se stabilise en partie avec la vie professionnelle.

On assiste depuis une génération à un brouillage des frontières entre les différentes étapes qui structurent l'avancement dans la jeunesse du fait notamment de la fin des grands rituels de passage profanes et religieux et probablement de l'allongement de la scolarité. Ceci explique pourquoi le terme de jeunesse est si flou. C'est pourquoi aussi les biens de consommation jouent probablement de plus en plus un rôle de mini marqueur de passage et d'appartenance en remplacement des rituels plus traditionnels. C'est souvent l'accumulation des micro-changements qui fait sens et signifie qu'une étape du cycle de vie est passée.

31 Chaque étape au sein du cycle de vie jeune marque bien un moment de la vie d'un jeune, mais les frontières entre deux étapes sont floues du fait de la diversité des trajectoires de vie scolaire et professionnelle. Il faut donc sans cesse réinterroger le contenu des marqueurs, la place des frontières et les moments de passage. C'est cette connaissance qui permet de comprendre la place d'une marque ou d'un produit tout au long du cycle de vie. C'est ce qui explique pour une part les modes de consommation par âge ou génération.

En résumé, le poids de la marque est variable en fonction de ces étapes. La marque sert à indiquer le passage entre deux étapes du cycle de vie ou entre deux appartenances sociales. Elle indique aussi l'appartenance à une étape du cycle de vie ou à un groupe. Elle signifie autant la distinction entre deux groupes ("Bourges » contre « racaille», « Mac » contre «PC») que l'exclusion sociale. C'est pourquoi la consommation est analysée ici comme un phénomène collectif qui signifie l'intégration ou l'exclusion sociale. Le rapport à la marque est bien un phénomène social mais qui relève d'une dynamique dans le temps qui explique en partie sa variabilité.

33 C'est pourquoi le rapport à la marque évolue en fonction du cycle de vie du produit depuis l'entreprise où il est produit, le magasin où il est distribué, l'espace domestique où il est utilisé et consommé jusqu'à sa séparation sous forme de déchet ou de don. Il évolue aussi en fonction du cycle de vie des personnes à une époque donnée, des effets de génération entre deux époques, des effets d'appartenance sociale liés aux positions dans l'espace urbain, des effets de genre hétéro ou homo, des effets culturels (les produits 
interdits ou permis en fonction des religions comme le voile avec l'Islam, les produits casher, etc.) et enfin des effets de réseau familial, amical et professionnel. C'est la complexité et la variabilité de ces facteurs qui expliquent d'un point de vue micro-social l'infidélité à la marque.

\section{BIBLIOGRAPHIE}

Alami S., Boissard A., Golfier A., Marnat D., Horn R., Desjeux D., Play and Learning in a Changing World (France, Germany, Austria, Switzerland, the United States and China), Paris, Interlis/Lego, 2004.

Bonnet M., La ville aux limites de la mobilité, Paris, PUF, à paraître.

Bouchardeau M., Nguyen E., Ouelhadj F., Suntherraswaran K., Le nouveau rapport à la marque chez les 25-35 ans, Magistère de sciences sociales, Paris 5, Sorbonne/TNSofres (sous la dir. de Perreau E. et Desjeux D.), 2004.

Bouly de Lesdain S., Lavon S., Desjeux D., L'argent en France. Pratiques et représentation du crédit à la consommation., Enquête exploratoire qualitative, Paris, CERLIS (CNRS, Sorbonne)/ COFIDIS, 2002.

Chwarzciannek J., «Stoppub», un mouvement anti-pub ?, Paris 5/Sorbonne (DEA sous la dir.de D. Desjeux), 2004.

Desjeux D., « Postface », in : Jean Michel Normand, Les nouveaux imaginaires de la consommation, Paris, PUF, 2004, pp. 191-208.

Desjeux D., Les sciences sociales, Paris, PUF, Que-sais-je ?, 2004.

Desjeux D., Taponier S., Jarvin M., Regard anthropologique sur les bars de nuit, Paris, l'Harmattan, 1998.

Desjeux D., Medina P., Berthier C., Pratiques et imaginaire de la consommation de pastis, Paris, Argonautes/Pernod Ricard, 1994.

Diasio N. (éd.), Au palais de Dame tartine. Regards européens sur la consommation enfantine, Paris, l'Harmattan, 2004.

Fanardjis L., Comportement vestimentaire d'adolescents Yvelinois, Paris, 5/Sorbonne (Maîtrise sous la dir.de D. Desjeux), 1997.

Galland O., Sociologie de la jeunesse, Paris, A. Colin, 1991.

Gamba T., Soin du corps au masculin, Paris 5/Sorbonne (Maîtrise sous la dir.de D. Desjeux), 1999.

Garabuau-Moussaoui I., "Jeunes et consommation », in Pugeault Catherine-Cicchelli, Cicchelli Vincenzo (éds.), Ce que nous savons des jeunes, Paris, PUF, 2004, pp 69-80.

Garabuau-Moussaoui I., Desjeux D. (éds.), Objet banal, objet social, Paris, l'Harmattan, 2003.

Garabuau-Moussaoui I., Cuisine et indépendances, Paris, L'Harmattan, 2002.

Gaulier X., La deuxième carrière, Paris, Seuil, 1988.

Guillemard A., L’âge de l'emploi. Les sociétés à l'épreuve du vieillissement, Paris, A. Colin, 2003. 
Guillot A., De l'adolescence au monde du travail : changer de tenue, Paris 5, Sorbonne (maîtrise sous la dir. de D. Desjeux), 2004.

LadwenR., Le comportement du consommateur et de l'acheteur, Economica, Paris (2ème édition), 2003.

Leveque C., Reflets aquatiques et expressions corporelles. Etudes des pratiques et de l'espace du corps dans la salle de bain, Paris 5, Sorbonne (maitrise sous la dir. de D. Desjeux), 1998.

Maffesoli M., Le temps des tribus, Paris, Méridiens Klinsieck, 1985.

Mathieu-Fritz A., Les huissiers de justice, Paris, PUF, 2005.

Normand JM., Les nouveaux imaginaires de la consommation, Paris, PUF, 2004.

Orfeuil JP. (éd.), Transports, pauvretés, exclusions. Pouvoir bouger pour s'en sortir, éd. De l'Aube, 2004.

Pugeault C., Cicchelli V. (éds.), Ce que nous savons des jeunes, Paris, PUF, 2004.

Roustan M. (éd.), La pratique du jeu vidéo : réalité ou virtualité ?, Paris, l'Harmattan, 2003.

\section{NOTES}

1. Cf. Desjeux D., Bouly de Lesdain S., Lavon S., L'argent en France. Pratiques et représentation du crédit à la consommation. Enquête exploratoire qualitative, Paris, CERLIS (CNRS, Sorbonne) / COFIDIS, 91 p (multig.) et Alexandre Mathieu-Fritz, 2005, Les huissiers de justice, Paris, PUF, 2002.

\section{RÉSUMÉS}

Le but de cet article est de montrer que l'infidélité du consommateur peut s'expliquer d'un point de vue socio-anthropologique par l'instabilité ou la variabilité de la vie sociale. Cette variabilité de la vie sociale est elle-même organisée autour des étapes du cycle de vie. A travers l'étude de produits comme l'alcool ou les vêtements, il est montré comment les jeunes s'autonomisent de la contrainte de leur parents tout en intégrant les normes de leur groupe de pair. Choisir ou refuser un produit et sa marque est lié à la variabilité des normes sociales. La marque ou son refus apparaît comme l'expression d'une contrainte forte, celle du groupe d'appartenance.

This article shows that, from a socio-anthropological point of view, a lack of consumer loyalty can be explained by instability or change in social life. This change is linked to the stages of the life course. An examination of young people's behaviors involving alcohol and clothing demonstrates how they are becoming more autonomous from their parents but more subject to the social norm of their peer group. Choosing - or not choosing - a product or a brand is directly linked to the variability of peer social norms. This choice indicates a strong social constraint, that of group affiliation. 


\section{AUTEUR}

\section{DOMINIQUE DESJEUX}

Dominique Desjeux est Anthropologue, Professeur d'Anthropologie sociale et culturelle à la Sorbonne, rédacteur en chef de la revue Consommations et Sociétés, et consultant international. Il s'intéresse notamment à l'anthropologie de la consommation et à l'ethno-marketing. Mail : d.desjeux@argonautes.fr http://www.argonautes.fr http:// www.consommations-societes.net 